UDC: 515.122

\title{
MORE ON $*_{*}$-CONNECTEDNESS
}

\author{
SELAHATTIN KILINÇ
}

\begin{abstract}
In [4 $*_{*}$-connected, $*$-cl-connected and $*-$ cl $^{*}$-connected ideal space are introduced and studied by Modak and Noiri. We further study the properties of these sets and give a characterization of $*_{*}$ connected ideal space.
\end{abstract}

The concept of ideal topological space has been studied by Kuratowski [3] Kuratowski and Vaidyanathswamy, [5]. A nonempty collection $I$ of subsets of $X$ is called an ideal in $X$ if it has the following properties:

(i) $A \in I$ and $B \subset A$ implies $B \in I$ and

(ii) $A \in I$ and $B \in I$ implies $A \cup B \in I$.

Given a topological space $(X, \tau)$ with an ideal $I$ in $X,(X, \tau, I)$ is an ideal topological space (we call it an ideal space). For a subset $A$ of $X$ the local function of $A$ is defined as follows, [3]:

$$
A^{*}(I, \tau)=\{x \in X \mid U \cap A \notin I\},
$$

for every $U \in \tau(x)$ where $\tau(x)=\{U \in \tau \mid x \in U\}$. We will make use of basic facts about the local function [2] without mentioning it explicitly. A Kuratowski closure operator $c l^{*}()$ for a topology $\tau^{*}(I, \tau)$, called the $*$ topology finer then $\tau$ is defined by $c l^{*}(A)=A \cup A^{*}(I, \tau)$, [5]. When there is no room for confusion, we simply write $A^{*}$ for $A^{*}(\tau, I)$ and $\tau^{*}$ for $\tau^{*}(I, \tau)$. $*_{*}$-connected, $*$-cl-connected and $*$-cl*-connected ideal spaces are introduced and studied by Modak and Noiri in [4. In this paper we further study the properties of these spaces.

If $A \subset X, \operatorname{cl} A$ and $\operatorname{int}(A)$ will denote the closure and interior of $A$ in $(X, \tau)$ and $i n t^{*}(A)$ will denote the interior of $A$ in $\left(X, \tau^{*}\right)$. Subsets of $X$ closed in $\left(X, \tau^{*}\right)$ are called $*$-closed sets. A subset $A$ of $X$ in ideal space $(X, \tau, I)$ is $*$-closed if and only if $A^{*} \subset A$, [2]. Nonempty subsets $A, B$ of an ideal space $(X, \tau, I)$ are called $*_{*}$-separated if $A^{*} \cap B=A \cap B^{*}=A \cap B=\emptyset$ and are called $*$-cl-separated (resp. $*$-cl ${ }^{*}$-separated) if $A^{*} \cap \operatorname{cl}(B)=c l(A) \cap$ $B^{*}=A \cap B=\emptyset\left(\right.$ resp. $\left.\quad A^{*} \cap c l^{*}(B)=c l^{*}(A) \cap B^{*}=A \cap B=\emptyset\right)$, [4].

2010 Mathematics Subject Classification. 54A05, 54D05.

Key words and phrases. $*_{*}$-connected, $*$-cl-connected, $*$-cl ${ }^{*}$-connected. 
A subset $A$ of an ideal space $(X, \tau, I)$ is called $*_{*}$-connected if $A$ is not the union of two $*_{*}$-separated sets in $(X, \tau, I)$ and is called $*$-cl-connected (resp. *-cl*-connected) if $A$ is not the union of two $*$-cl-separated (resp. *-cl*-separated sets in $(X, \tau, I))$, [4].

\section{Main Results}

Lemma 1. 4 Let $(X, \tau, I)$ be an ideal space. If $A$ is a $*_{*}$-connected set of $X$ and $H, G$ are $*_{*}$-separated sets of $X$ with $A \subset H \cup G$, then either $A \subset H$ or $A \subset G$.

Lemma 2. 4] Let $(X, \tau, I)$ be an ideal space. If $A$ is a $*$-cl-connected subset of $X$ and $H, G$ are $*$-cl-separated sets of $X$ with $A \subset H \cup G$, then either $A \subset H$ or $A \subset G$.

Lemma 3. 4. Let $(X, \tau, I)$ be an ideal space. If $A$ is a*-c $*^{*}$-connected subset of $X$ and $H, G$ are $*-c l^{*}$-separated sets of $X$ with $A \subset H \cup G$, then either $A \subset H$ or $A \subset G$.

Theorem 1. Let $(X, \tau, I)$ be an ideal space. If $A$ and $B$ are nonempty disjoint sets such that $A$ and $B$ are $*$-open, then $A$ and $B$ are $*_{*}$-separated.

Proof. Let $A$ and $B$ be nonempty disjoint sets such that $A \cap B=\emptyset$. We have $A \subset X-B$ and so $c l^{*}(A) \subset c l^{*}(X-B)=X-B$. Always $A^{*} \subseteq$ $c l^{*}(A) \subset X-B$ which implies that $A^{*} \cap B=\emptyset$. Again $B \subset X-A$ which implies that $A \cap B^{*}=\emptyset$. So, $A^{*} \cap B=A \cap B^{*}=A \cap B=\emptyset$ and therefore $A$ and $B$ are $*_{*}$-separated.

Theorem 2. Let $A$ and $B$ be two *-cl-separated sets in an ideal space $(X, \tau, I)$. If $C$ and $D$ are nonempty subsets such that $C \subset A$ and $D \subset B$, then $C$ and $D$ are also $*$-cl-separated.

Proof. Since $A$ and $B$ are $*$-cl-separated, $A^{*} \cap c l(B)=c l(A) \cap B^{*}=A \cap B=$ $\emptyset$. We know that, since $C \subset A$ and $D \subset B$, then $\operatorname{cl}(C) \subset \operatorname{cl}(A), \operatorname{cl}(D) \subset$ $\operatorname{cl}(B)$ and $C^{*} \subset A^{*}, D^{*} \subset B^{*}$ such that $C^{*} \cap \operatorname{cl}(D) \subset A^{*} \cap \operatorname{cl}(B)=\emptyset$ and so $C^{*} \cap \operatorname{cl}(D)=\emptyset$. Similarly we can prove that $\operatorname{cl}(C) \cap D^{*}=\emptyset$. Hence, $C^{*} \cap \operatorname{cl}(D)=\operatorname{cl}(C) \cap D^{*}=C \cap D=\emptyset$. Consequently, $C$ and $D$ are *-cl-separated.

Theorem 3. Let $A$ and $B$ be two $*_{*}$-separated (resp. *-cl*-separated) sets in an ideal space $(X, \tau, I)$. If $C$ and $D$ are nonempty subsets such that $C \subset A$ and $D \subset B$, then $C$ and $D$ are also $*_{*}$-separated (resp. $*$-cl*-separated).

Proof. The proof is similar with the proof of Theorem 2 .

Theorem 4. Let $(X, \tau, I)$ be an ideal space and $A, B \subset X$. If $A$ and $B$ are *-open or $*$-closed, then $A-B$ and $B-A$ are $*_{*}$-separated. 
Proof. $(A-B)^{*} \cap(B-A) \subseteq(A \cap(X-B))^{*} \cap(B-A) \subset A^{*} \cap(X-B)^{*} \cap$ $(B \cap(X-A))=A^{*} \cap(X-A) \cap(X-B)^{*} \cap B$. If $A$ is $*$-closed, then $A^{*} \subset A$, such that $A^{*} \cap(X-A) \cap(X-B)^{*} \cap B=\emptyset$. If $B$ is *-open, $(X-B)$ is *closed, then $(X-B)^{*} \subset(X-B)$, such that $A^{*} \cap(X-A) \cap(X-B)^{*} \cap B=\emptyset$. Similarly, we can show that $(A-B) \cap(B-A)^{*}=\emptyset$. Consequently, $A-B$ and $B-A$ are $*_{*}$-separated.

Theorem 5. Let $a$ and $b$ be distinct points of a subset $C$ of an ideal space $(X, \tau, I)$. If they are elements of some $*_{*}$-connected subset of $C$, then $C$ is $a *_{*}$-connected subset of $X$.

Proof. Suppose $C$ is not $*_{*}$-connected. Then there exist nonempty subsets $A$ and $B$ of $X$ such that $A^{*} \cap B=A \cap B^{*}=A \cap B=\emptyset$ and $C=A \cup B$. Since $A$ and $B$ are nonempty sets there exists a point $a \in A$ and a point $b \in B$. By hypothesis, $a$ and $b$ must be elements of a $*_{*}$-connected subset $E$ of $C$. Since $E \subset A \cup B$, by Lemma 1, either $E \subset A$ or $E \subset B$. Consequently, either $a$ and $b$ are both in $A$ or both in $B$. Let's say that $a$ and $b$ are elements of the set $A$. Then $A \cap B \neq \emptyset$, that is a contradiction to the fact that $A$ and $B$ are disjoint. Therefore, $C$ must be $*_{*}$-connected.

Theorem 6. Let $a$ and $b$ be distinct points of a subset $C$ of an ideal space $(X, \tau, I)$. If they are elements of some $*$-cl-connected (resp. $*$-cl $l^{*}$-connected) subset of $C$, then $C$ is a $*$-cl-connected (resp. *-cl*-connected) subset of $X$.

Proof. The proof is similar with the proof of Theorem 5.

Theorem 7. Let $(X, \tau, I)$ be a $*_{*}$-connected ideal topological space. If $A$ is $a *_{*}$-connected subset of $(X, \tau, I)$ and $X-A$ is a union of two $*_{*}$-separated sets $B$ and $C$, then $A \cup B$ and $A \cup C$ are $*_{*}$-connected.

Proof. Suppose that $A \cup B$ is not $*_{*}$-connected. Then there exist two nonempty $*_{*}$-separated sets $G$ and $H$, such that $A \cup B=G \cup H$. Since $A$ is a $*_{*}$-connected, $A \subset A \cup B=G \cup H$. From Lemma 2 we know either $A \subset H$ or $A \subset G$. Suppose $A \subset G$. Since $A \cup B=G \cup H, A \subset G$ implies that $A \cup B \subset G \cup B$ and so $G \cup H \subset G \cup B$. Hence, $H \subset B$. Since $B$ and $C$ are $*_{*}$-separated, $H$ and $C$ are also $*_{*}$-separated. Thus $H$ is $*_{*^{-}}$ separated from $G$ as well as $C$. Now, $H^{*} \cap(G \cup C)=\left(H^{*} \cap G\right) \cup\left(H^{*} \cap C\right)=\emptyset$, $H \cap(G \cup C)^{*}=H \cap\left(G^{*} \cup C^{*}\right)=\left(H \cap G^{*}\right) \cup\left(H \cap C^{*}\right)=\emptyset$ and $H \cap(G \cup C)=$ $(H \cap G) \cup(H \cap C)=\emptyset$. Therefore, $H$ is $*_{*}$-separated from $G \cup C$. Since $X-A=B \cup C, X=A \cup(B \cup C)=(A \cup B) \cup C=(G \cup H) \cup C$, $A \cup B=G \cup H$ and so $X=(G \cup C) \cup H$. Thus, $X$ is the union of two nonempty $*_{*}$-separated sets $G \cup C$ and $H$, which is a contradiction. Similarly, contradiction will arise if $A \subset H$. Hence $A \cup B$ is $*_{*}$-connected. One can prove in a similar way that $A \cup C$ is $*_{*}$-connected. 
Theorem 8. If $A$ is a*-cl-connected $(*$-cl*-connected) subset of a*-clconnected (*-cl*-connected) ideal topological space $(X, \tau, I)$ such that $X-A$ is a union of two $*$-cl-separated (*-cl*-separated) sets $B$ and $C$ then $A \cup B$ and $A \cup C$ are $*$-cl-connected (*-cl*-connected).

Proof. The proof is similar with the proof of Theorem 7 .

Theorem 9. Let $A$ and $B$ be two $*_{*}$-connected sets of an ideal space $(X, \tau, I)$. If they are not $*_{*}$-separated, then $A \cup B$ is $*_{*}$-connected.

Proof. Let $A$ and $B$ be $*_{*}$-connected in $X$. Suppose $A \cup B$ is not $*_{*}$ connected. Then, there exist two nonempty disjoint $*_{*}$-separated sets $G$ and $H$ such that $A \cup B=G \cup H$. Since $A$ and $B$ are $*_{*}$-connected by Lemma 1 either $A \subset G$ and $B \subset H$ or $B \subset G$ and $A \subset H$. Now, if $A \subset G$ and $B \subset H$, then $A \cap H=B \cap G=\emptyset$. Therefore $(A \cup B) \cap G=$ $(A \cap G) \cup(B \cap G)=(A \cap G) \cup \emptyset=(A \cap G)=A$. Also, $(A \cup B) \cap$ $H=(A \cap H) \cup(B \cap H)=\emptyset \cup(B \cap H)=(B \cap H)=B$. Similarly, if $B \subset G$ and $A \subset H$ then $(A \cup B) \cap G=A$ and $(A \cup B) \cap H=B$. Now, $((A \cup B) \cap H) \cap((A \cup B) \cap G)^{*} \subset((A \cup B) \cap H) \cap\left((A \cup B)^{*} \cap G^{*}\right)=$ $(A \cup B) \cap H \cap G^{*} \cap(A \cup B)^{*}=\emptyset,((A \cup B) \cap H)^{*} \cap((A \cup B) \cap G) \subset$ $\left((A \cup B)^{*} \cap H^{*}\right) \cap((A \cup B) \cap G)=(A \cup B)^{*} \cap H^{*} \cap G \cap(A \cup B)=\emptyset$ and $((A \cup B) \cap H) \cap((A \cup B) \cap G)=((A \cup B) \cap H \cap G \cap(A \cup B)=\emptyset$. Therefore, $(A \cup B) \cap G$ and $(A \cup B) \cap H$ are $*_{*}$-separated. Thus, $A$ and $B$ are $*_{*}$-separated which is a contradiction. Hence, $A \cup B$ is $*_{*}$-connected.

Theorem 10. Let $A$ and $B$ be two *-cl-connected sets of an ideal space $(X, \tau, I)$. If none of them is $*$-cl-connected, then $A \cup B$ is $*$-cl-connected.

Proof. Let $A$ and $B$ be $*$-cl-connected sets in $X$. Suppose $A \cup B$ is not $*$-cl-connected. Then, there exist two nonempty disjoint $*$-cl-separated sets $G$ and $H$ such that $A \cup B=G \cup H$. Since $A$ and $B$ are $*$-cl-connected, by Lemma 1 either $A \subset G$ and $B \subset H$ or $B \subset G$ and $A \subset H$. Now, if $A \subset G$ and $B \subset H$, then $A \cap H=B \cap G=\emptyset$. Therefore $(A \cup B) \cap G=$ $(A \cap G) \cup(B \cap G)=(A \cap G) \cup \emptyset=(A \cap G)=A$. Also $(A \cup B) \cap$ $H=(A \cap H) \cup(B \cap H)=\emptyset \cup(B \cap H)=(B \cap H)=B$. Similarly, if $B \subset G$ and $A \subset H$, then $(A \cup B) \cap G=A$ and $(A \cup B) \cap H=B$. Now, $((A \cup B) \cap H)^{*} \cap \operatorname{cl}((A \cup B) \cap G) \subset\left((A \cup B)^{*} \cap H^{*}\right) \cap \operatorname{cl}(A \cup B) \cap c l G=$ $(A \cup B)^{*} \cap \operatorname{cl}(A \cup B) \cap H^{*} \cap c l G=\emptyset, c l((A \cup B) \cap H) \cap((A \cup B) \cap G)^{*} \subset$ $\operatorname{cl}(A \cup B) \cap \operatorname{cl} H \cap(A \cup B)^{*} \cap G^{*}=\operatorname{cl}(A \cup B) \cap(A \cup B)^{*} \cap \operatorname{cl} H \cap G^{*}=\emptyset$ and $((A \cup B) \cap H) \cap((A \cup B) \cap G)=((A \cup B) \cap H \cap G \cap(A \cup B)=\emptyset$. Therefore, $(A \cup B) \cap G$ and $(A \cup B) \cap H$ are $*$-cl-separated. Thus, $A$ and $B$ are *-cl-separated which is a contradiction. Hence, $A \cup B$ is $*$-cl-connected.

Theorem 11. If $A$ and $B$ are $*-c l^{*}$-connected sets of an ideal space $(X, \tau, I)$ such that none of them is $*$-cl*-separated, then $A \cup B$ is $*$-cl*-connected. 
Proof. The proof is similar with the proof of Theorem 10 .

The following example shows that the union of two $*_{*}$-connected, (resp. $*$-cl-connected, $*$ - $c l{ }^{*}$-connected) sets is not a $*_{*}$-connected set, (resp. $*$-clconnected, $*-c l^{*}$-connected sets). However, Theorems 9, 10 and 11 show that the union of two $*_{*}$-connected (resp. *-cl-connected, $*$-cl*-connected sets is a $*_{*}$-connected, (resp. $*-c l$-connected, $*-c l^{*}$-connected) set if none of them is $*_{*}$-separated (resp. $*-c l$-separated, $*-c l^{*}$-separated).

Example 1. Let $(X, \tau, I)$ be ideal space, where $X=\{a, b, c, d\}, \tau=$ $\{\varnothing,\{b\},\{b, c\},\{a, b, d\}, X\}$ and $I=\{\varnothing,\{b\}\}$. If $A=\{a, b\}, B=\{a, d\}$, then $A$ and $B$ are $*_{*}$-connected, but $A \cup B$ is not.

In what follows, $B d(A)$ denotes the of boundary $A$.

Theorem 12. Let $(X, \tau, I)$ be an ideal space and $A \subset X$. If $C$ is $*_{*}$ connected subspace of $X$ that intersect both $A$ and $X-A$, then $C$ intersects $B d(A)$.

Proof. Suppose $C \cap B d(A)=\emptyset$. So, $B d(A)=(\operatorname{cl}(A) \cap \operatorname{cl}(X-A))$ and $C \cap(\operatorname{cl}(A) \cap \operatorname{cl}(X-A))=\emptyset$. Now, $C=C \cap X=C \cap(A \cup(X-A))=$ $(C \cap A) \cup(C \cap(X-A))$. Also, we know that $(C \cap A)^{*} \cap(C \cap(X-A)) \subset$ $\left(C^{*} \cap A^{*}\right) \cap(C \cap(X-A))=C \cap C^{*} \cap\left(A^{*} \cap(X-A)\right)$. So, $A^{*} \subset \operatorname{cl}(A)$ and $(X-A) \subset \operatorname{cl}(X-A)=\emptyset$, which implies that $C^{*} \cap C \cap\left(A^{*} \cap(X-A)\right)=\emptyset$. Then $(C \cap A) \cap(C \cap(X-A))^{*} \subset(C \cap A) \cap\left(C^{*} \cap(X-A)^{*}\right)=C \cap C^{*} \cap$ $\left(A \cap(X-A)^{*}\right)=\emptyset$ and $(C \cap A) \cap(C \cap(X-A))=C \cap A \cap(X-A)=\emptyset$. Thus, $(C \cap A)$ and $(C \cap(X-A))$ form a $*_{*}$-separation for $C$, which is a contradiction. Hence, $C \cap B d(A) \neq \emptyset$.

Theorem 13. Let $(X, \tau, I)$ be an ideal space and $A \subset X$. If $C$ is $*$-clconnected (resp. *-cl*-connected) subspace of $X$ that intersect both $A$ and $X-A$, then $C$ intersects $B d(A)$.

Proof. The proof is similar with the proof of Theorem 12 .

Theorem 14. Let $(X, \tau, I)$ be an ideal space. Both $*$-closed or *-open separated sets in this space are $*_{*}$-separated.

Proof. A and B are separated sets, $A \cap B=\emptyset$. If $A$ and $B$ are $*$-closed, then $A^{*} \subset A$ and $B^{*} \subset B$, so that $A^{*} \cap B=A \cap B^{*}=A \cap B=\emptyset$. We will prove that $A$ and $B$ are $*_{*}$-separated. If $A$ and $B$ are $*$-open, then $X-A$ and $X-B$ are $*$-closed. So, $A \subset X-B$ which implies that $A^{*} \subset(X-B)^{*} \subset X-B$, and $B \subset X-A$ which implies that $B^{*} \subset(X-A)^{*} \subset X-A$. We obtain that $A^{*} \cap B=(X-B) \cap B=\emptyset$ and $A \cap B^{*}=A \cap(X-A)=\emptyset$. Consequently, $A$ and $B$ are $*_{*}$-separated. 
Theorem 15. Let $(X, \tau, I)$ be an ideal space and $A, B \subset X$. If $c l^{*}(A) \cap B=$ $\emptyset$ and $A \cup B$ is *-closed, then $A$ is $*$-closed set.

Proof. So $A \cup B$ is $*$-closed, that implies $c l^{*}(A \cup B)=c l^{*}(A) \cup c l^{*}(B)=A \cup B$ and $c l^{*}(A) \subset A \cup B$. If $c l^{*}(A) \cap B=\emptyset$, then $c l^{*}(A) \subset A$. We know that $A \subset c l^{*}(A)$ and thus $A=c l^{*}(A)$. So, $A$ is $*$-closed.

Theorem 16. Let $(X, \tau, I)$ be an ideal space and $A, B \subset X$. If $A \cap c l^{*}(B)=$ $\emptyset$ and $A \cup B$ is *-open, then $A$ is *-open set.

Proof. If $A \cap c l^{*}(B)=\emptyset$, then $A \subset\left(X-c l^{*}(B)\right)$. Since $A \cup B$ is *-open, then $(A \cup B) \cap\left(X-c l^{*}(B)\right)=\left(A \cap\left(X-c l^{*}(B)\right)\right) \cup\left(B \cap\left(X-c l^{*}(B)\right)\right)$ is a $*$-open set. So, $A=\left(A \cap\left(X-c l^{*}(B)\right)\right) \cup\left(B \cap\left(X-c l^{*}(B)\right)\right)$ is a *-open set.

Corollary 16.1. Let $(X, \tau, I)$ be an ideal space and $A, B \subset X$. If $A$ and $B$ are not $*-c l$-connected (resp. $*-c l^{*}$-connected) and $A \cup B$ is $*$-closed, then $A$ and $B$ are *-closed; if $A \cup B$ is *-open, then $A$ and $B$ are *-open.

Tag1, Bhardwaj and Singh, in [1] introduced a relationship between continuousness and $C l^{*}$-connectedness and $C L-C l^{*}$-connectedness. Now we will examine the relationship between continuousness and $*_{*}$-connected, (resp. *-cl-connected, $*$-cl ${ }^{*}$-connected) ideal space.

Definition 1. [1] A function $f:\left(X, \tau_{1}, I_{1}\right) \rightarrow\left(X, \tau_{2}, I_{2}\right)$ is said to be:

(1) continuous if the inverse image of each open set in $Y$ under $f$ is open in $X$;

(2) contra-continuous if the inverse image of each open set in $Y$ under $f$ is closed in $X$;

(3) $\tau_{1}-\tau_{2^{*}}$-continuous if the inverse image of each $*$-open set in $Y$ under $f$ is open in $X$;

(4) $\tau_{1^{*}}-\tau_{2^{*}-\text { continuous }}$ if the inverse image of each $*$-open set in $Y$ under $f$ is $*$-open in $X$;

(5) $\tau_{1}-\tau_{2^{*}}$-contra-continuous if the inverse image of each $*$-open set in $Y$ under $f$ is closed in $X$;

(6) $\tau_{1^{*}}-\tau_{2^{*} \text {-contra-continuous }}$ if the inverse image of each $*$-open set in $Y$ under $f$ is $*$-closed in $X$.

Theorem 17. 1] Let $f:\left(X, \tau_{1}, I_{1}\right) \rightarrow\left(Y, \tau_{2}, I_{2}\right)$ be a continuous surjection. If $X$ is $*_{*}$-connected, then $Y$ is connected.

Proof. Suppose that $Y$ is not connected. So, there is a nonempty clopen proper subset $A$ of $Y$. Then $f^{-1}(A)$ is nonempty clopen subset of $X$ and hence, $f^{-1}(A)$ and $X-f^{-1}(A)$ are disjoint. We know that $A^{*} \subset c l^{*}(A) \subset$ $\operatorname{cl}(A)$. So, $f^{-1}(A)$ is clopen since $\left(f^{-1}(A)\right)^{*} \cap\left(X-f^{-1}(A)\right)=\left(f^{-1}(A)\right) \cap$ $\left(X-f^{-1}(A)\right)^{*}=\emptyset$, i.e. $X$ is not $*_{*}$-connected, that is a contradiction. 
Theorem 18. Let $f:\left(X, \tau_{1}, I_{1}\right) \rightarrow\left(Y, \tau_{2}, I_{2}\right)$ be a continuous surjection. If $X$ is resp. *-cl-connected, $*$-cl*-connected, then $Y$ is connected.

Proof. The proof is similar with the proof of Theorem 17 .

Theorem 19. Let $f:\left(X, \tau_{1}, I_{1}\right) \rightarrow\left(Y, \tau_{2}, I_{2}\right)$ be a continuous surjection. If $X$ is $*_{*}$-connected, then $Y$ is $*_{*}$-connected.

Proof. Suppose that $Y$ is not $*_{*}$-connected. Then there are nonempty subsets $A$ and $B$ of $Y$ and hence $Y=A \cup B, A^{*} \cap B=A \cap B^{*}=A \cap B=\emptyset$. From the fact that $f$ is a continuous surjection, $X=f^{-1}(A) \cup f^{-1}(B)$ and $f^{-1}(A), f^{-1}(B)$ are clopen that $\left(f^{-1}(A)\right)^{*} \cap f^{-1}(B)=f^{-1}(A) \cap$ $\left(f^{-1}(B)\right)^{*}=f^{-1}(A) \cap f^{-1}(B)=\emptyset$. We proved that $X$ is not $*_{*}$-connected, that is a contradiction.

Theorem 20. Let $f:\left(X, \tau_{1}, I_{1}\right) \rightarrow\left(Y, \tau_{2}, I_{2}\right)$ be a continuous surjection. If $X$ is resp. *-cl-connected, $*-c l^{*}$-connected, then $Y$ is resp. *-clconnected, $*-c l^{*}$-connected.

Proof. The proof is similar with the proof of Theorem 19 .

Theorem 21. Let $f:\left(X, \tau_{1}, I_{1}\right) \rightarrow\left(Y, \tau_{2}, I_{2}\right)$ be a contra-continuous surjection. If $X$ is $*_{*}$-connected, then $Y$ is connected.

Proof. Suppose that $Y$ is not connected. Then there are nonempty subsets $A$ and $B$ of $Y$ and hence $Y=A \cup B, A^{*} \cap B=A \cap B^{*}=A \cap B=\emptyset$. From the fact that $f$ is contra-continuous surjection, $X=f^{-1}(A) \cup f^{-1}(B)$ and $f^{-1}(A), f^{-1}(B)$ are clopen that $\left(f^{-1}(A)\right)^{*} \cap f^{-1}(B)=f^{-1}(A) \cap$ $\left(f^{-1}(B)\right)^{*}=f^{-1}(A) \cap f^{-1}(B)=\emptyset$. We proved that $X$ is not $*_{*}$-connected, that is a contradiction.

Theorem 22. Let $f:\left(X, \tau_{1}, I_{1}\right) \rightarrow\left(Y, \tau_{2}, I_{2}\right)$ be a contra-continuous surjection. If $X$ is $*-c l$-connected, $*-c l^{*}$-connected, then $Y$ is $*-c l$-connected, $*-c l^{*}$-connected.

Proof. The proof is similar with the proof of Theorem 21 .

Theorem 23. Let $f:\left(X, \tau_{1}, I_{1}\right) \rightarrow\left(Y, \tau_{2}, I_{2}\right)$ be a $\tau_{1^{*}}-\tau_{2^{*}-\text { continuous }}$ surjection. If $X$ is $*_{*}$-connected, then $Y$ is $*_{*}$-connected.

Proof. Suppose that $Y$ is not $*_{*}$-connected. Then there are nonempty subsets $A$ and $B$ of $Y$ and hence $Y=A \cup B, A^{*} \cap B=A \cap B^{*}=A \cap B=$ $\emptyset$. Since $f$ is $\tau_{1^{*}}-\tau_{2^{*} \text {-continuous surjection, then } A \text { and } B \text { are } * \text {-open }}$ sets in $Y$ so that $f^{-1}(A), f^{-1}(B)$ are $*$-open and $*$-closed in $X$ and so $\left(f^{-1}(A)\right)^{*} \cap f^{-1}(B)=f^{-1}(A) \cap\left(f^{-1}(B)\right)^{*}=f^{-1}(A) \cap f^{-1}(B)=\emptyset$. Hence, $X$ is not $*_{*}$-connected, that is a contradiction. 
Theorem 24. Let $f:\left(X, \tau_{1}, I_{1}\right) \rightarrow\left(Y, \tau_{2}, I_{2}\right)$ be a $\tau_{1^{*}}-\tau_{2^{*} \text {-continuous }}$ surjection. If $X$ is $*-c l^{*}$-connected, then $Y$ is $*-c l^{*}$-connected.

Proof. The proof is similar with the proof of Theorem 23

Theorem 25. Let $f:\left(X, \tau_{1}, I_{1}\right) \rightarrow\left(Y, \tau_{2}, I_{2}\right)$ be a $\tau_{1^{*}}-\tau_{2^{*}}$-contra-continuous surjection. If $X$ is $*_{*}$-connected and $*-c l^{*}$-connected, then $Y$ is $*_{*}$-connected and $*-c l^{*}$-connected.

Proof. The proof is similar with the proof of Theorem 23

Theorem 26. Let $f:\left(X, \tau_{1}, I_{1}\right) \rightarrow\left(Y, \tau_{2}, I_{2}\right)$ be a $\tau_{1}-\tau_{2^{*} \text {-continuous }}$ surjection. If $X$ is $*_{*}$-connected, then $Y$ is $*_{*}$-connected.

Proof. We know that $\tau_{1} \subset \tau_{1}^{*}$ and $\tau_{2} \subset \tau_{2}^{*}$. If $f:\left(X, \tau_{1}, I_{1}\right) \rightarrow\left(Y, \tau_{2}, I_{2}\right)$ is

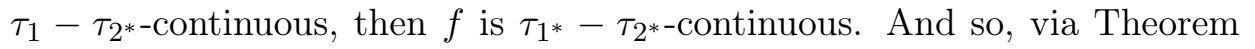
25. $Y$ is $*_{*}$-connected.

Theorem 27. Let $f:\left(X, \tau_{1}, I_{1}\right) \rightarrow\left(Y, \tau_{2}, I_{2}\right)$ be a $\tau_{1}-\tau_{2^{*} \text {-continuous }}$ surjection. If $X$ is $*-c l^{*}$-connected, then $Y$ is $*-c l^{*}$-connected.

Proof. The proof is similar with the proof of Theorem 26 .

Theorem 28. Let $f:\left(X, \tau_{1}, I_{1}\right) \rightarrow\left(Y, \tau_{2}, I_{2}\right)$ be a $\tau_{1}-\tau_{2^{*}-\text { contra-continuous }}$ surjection. If $X$ is $*_{*}$-connected and $*-c l^{*}$-connected, then $Y$ is $*_{*}$-connected and $*-c l^{*}$-connected.

Proof. The proof is similar with the proof of Theorem 26.

\section{REFERENCES}

[1] B.K. Tagı, M. Bhardwaj and S. Singh, $C l^{*}$-connectedness and $C L-C l^{*}$-connectedness in ideal topological space, Mat. Bilten, 42 (LXVIII) 2 (2018), 91-100.

[2] D. Jankovic and T.R. Hamlett, New topologies from old via ideals, Amer. Math. Monhtly 97 (1990), 295-310.

[3] K. Kuratowski, Topology, Vol I. New York, Academic press, 1996.

[4] S. Modak and T. Noiri, Connectedness of ideal topological spaces, Filomat 29 (4) (2015), 661-665 DOI 10.2298/FIL1504661M

[5] V. Vaidyanathaswamy, The localization theory in set topology, Proc. Indian Acad. Sci. 20 (1945), 51-61.

Department of Mathematics,

Faculty of Science and Literature

UNIVERSITY OF ÇUKUROVA

TURKEY

E-mail address: selahattinkilinc@hotmail.com 